\title{
Химический сдвиг и энергия обменного взаимодействия $1 s$ состояний доноров магния в кремнии. Возможность стимулированного излучения
}

\author{
(C) В.Н. Шастин ${ }^{1}$, Р.Х. Жукавин ${ }^{1}$, К.А. Ковалевский ${ }^{1, \uparrow}$, В.В. Цыпленков ${ }^{1}$, В.В. Румянцев ${ }^{1}$, \\ Д.В. Шенгуров ${ }^{1}$, С.Г. Павлов ${ }^{2}$, В.Б. Шуман ${ }^{3}$, Л.М. Порцель ${ }^{3}$, А.Н. Лодыгин ${ }^{3}$, \\ Ю.А. Астров ${ }^{3}$, Н.В. Абросимов ${ }^{4}$, J.M. Klopf ${ }^{5}$, H.-W. Hübers ${ }^{2,6}$ \\ ${ }^{1}$ Институт ффизики микроструктур Российской академии наук, \\ 607680 Нижний Новгород, Россия \\ ${ }^{2}$ Institute of Optical Sensor Systems, German Aerospace Center (DLR), \\ 12489 Berlin, Germany \\ ${ }^{3}$ Физико-технический институт им. А.Ф. Иоффе Российской академии наук, \\ 194021 Санкт-Петербург, Россия \\ ${ }^{4}$ Leibniz-Institut für Kristallzüchtung (IKZ), \\ 12489 Berlin, Germany \\ ${ }^{5}$ Helmholz-Zentrum Dresden-Rossendorf, \\ 01328 Dresden, Germany \\ ${ }^{6}$ Department of Physics, Humboldt-Universität zu Berlin, \\ 12489 Berlin, Germany \\ ฯ E-mail: atan4@yandex.ru
}

Поступила в Редакцию 24 апреля 2019 г.

В окончательной редакции 29 апреля 2019 г.

Принята к публикации 29 апреля 2019 г.

Представлены результаты экспериментов по обнаружению отщепленных состояний $1 \mathrm{~s}$, что позволяет определить химический сдвиг и энергию обменного взаимодействия нейтрального донора магния в кремнии. Положение парасостояний $1 s(E), 1 s\left(T_{2}\right)$, а также $2 s\left(A_{1}\right)$ определяет возможность получения инверсии населенности и конкретный механизм вынужденного комбинационного рассеяния света. Энергия парасостояния $1 s\left(T_{2}\right)$ определялась по положению резонансов Фано в спектре фотопроводимости $\mathrm{Si}: \mathrm{Mg}$ при $T=4 \mathrm{~K}$, а энергии ортосостояний $1 s\left(T_{2}\right), 1 s(E)$ - из спектров пропускания при повышенных температурах. На основе полученных экспериментальных данных сделаны оценки скоростей релаксации и проведен анализ возможных механизмов стимулированного излучения.

Ключевые слова: нейтральный двойной донор, магний, спектроскопия, резонанс Фано, фотопроводимость, инверсия населенностей, вынужденное комбинационное рассеяние света.

DOI: $10.21883 /$ FTP.2019.09.48135.18

\section{1. Введение}

Результаты по стимулированному излучению в терагерцовом диапазоне частот при оптическом возбуждении доноров V группы в кремнии [1] дают надежду на расширение диапазона излучения за счет использования новых примесей, у которых энергии переходов типа $2 p \rightarrow 1 s$ заметно отличаются. Одними из кандидатов на роль активных центров являются гелиоподобные центры [2] в кремнии, так как в них отщепленные состояния 1s имеют более глубокую энергию залегания, в отличие от доноров V группы [3]. Литературные данные относительно энергий парасостояния $1 s(E)$ в магнии указывали на величинур $\sim 52$ мэВ [4], что позволяло рассчитывать на создание инвертированного распределения между состояниями $2 p_{0} \rightarrow 1 s(E)$ и генерацию на частоте $\sim 10$ ТГц при фотовозбуждении среды. Однако существенные отличия гелиоподобных центров от мелких доноров в кремнии потребовали нового анализа механизмов формирования инверсии. В первую очередь это связано с наличием двух лестниц состояний (рис. 1,a), определяемых величиной полного спина системы двух электронов - спин-синглетной (парасостояния) и спинтриплетной (ортосостояния). Разница энергий между однотипными состояниями в этих лестницах определяется величиной обменного взаимодействия в двухзарядных примесях. При отсутствии спин-орбитального взаимодействия эти группы состояний не связаны оптически, и переходы с излучением/поглощением фононов между ними также отсутствуют. Кроме того, в двухзарядных донорах снимается вырождение по орбитальному моменту атома, вследствие чего $2 s$ состояния теперь могут лежать ниже $2 p_{0}$. Последнее приводит к появлению новых каналов релаксации из состояния $2 p_{0}$, что существенно изменяет скорость его релаксации. С другой стороны, наличие двух лестниц слабосвязанных состояний может приводить к возможности различных спин-зависимых эффектов, что расширяет спектр возможных приложений таких центров в полупроводниках.

Расчеты темпов безызлучательной релаксации возбужденных состояний показали возможность наличия коротких $\left(\sim 10^{-11} \mathrm{c}\right)$ релаксационных времен для переходов 


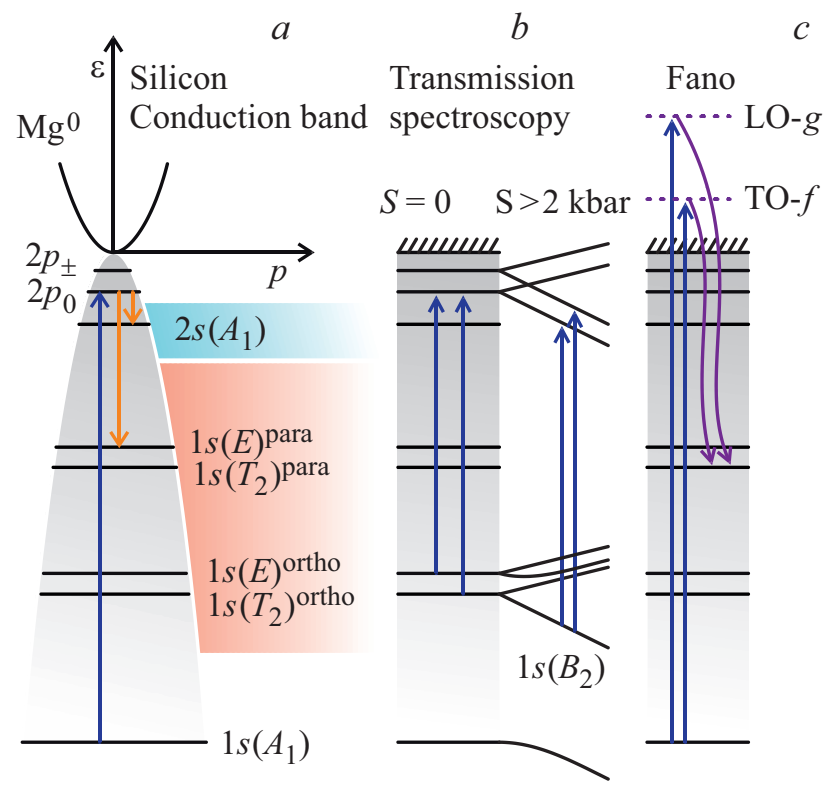

Pис. 1. $a-$ схема уровней нейтрального магния в кремнии, определяющих возможность создания источника стимулированного излучения на эффекте ВКР или инверсии населенности; $b-$ спектроскопия поглощения; $c$ - резонансы Фано.

с участием $1 s$ и $2 p$ состояний, что может усложнить достижение приемлемых уровней усиления в $\mathrm{Si}: \mathrm{Mg}$. В этом контексте важным оказывается нахождение положения нижележащих состояний $2 s\left(A_{1}\right)$ и $1 s\left(E, T_{2}\right)$, принадлежащих к группе парасостояний, которые на данный момент либо неизвестны, либо имеются только косвенные данные [3]. Настоящая работа ставит своей целью экспериментальное определение положения отщепленных $s$-состояний, определяемых химическим сдвигом и величиной обменного взаимодействия в доноpax магния, с последующим анализом релаксационных процессов и возможности получения стимулированного эффекта.

\section{2. Эксперимент}

Образцы кремния, легированного магнием методом диффузии [5], были ориентированы вдоль кристаллографических осей типа $\{001\}$ и отполированы. Для измерения фотопроводимости наносились контакты Ti/Au. Образцы $\mathrm{Si}: \mathrm{Mg}$ характеризовались путем измерения примесного поглощения при низких температурах (около $5 \mathrm{~K}$ ) с использованием фурье-спектрометра (Bruker Vertex 80v) со спектральным разрешением $0.1 \mathrm{~cm}^{-1}$. Это позволило установить значения концентрации $\mathrm{Mg}$ для различных образцов $\left(N_{D} \sim(1-3) \cdot 10^{15} \mathrm{~cm}^{-3}\right)$ и были определены концентрации других примесей и соответствующих комплексов магния. В эксперименте измерялись спектры пропускания при различных температурах $(6-200 \mathrm{~K})$, а также спектры фотопроводимости при температуре жидкого гелия. Так как спектроскопия неде- формированного кристалла не позволяет однозначно связать положение спектральной линии с конкретным переходом, в дополнение мы использовали одноосносжатые образцы. Для этого образцы монтировались в специальные модули фиксированного одноосного давления, которые помещались в соответствующие оптические криостаты.

\section{3. Результаты}

На рис. 2 представлены спектры пропускания для кремния, легированного донорами магния при различных температурах, при приложении одноосного давления (более 2 кбар) к образцу вдоль кристаллографического направления [001]. Одноосное сжатие приводит к междолинному расщеплению, и, так как состояние $1 s(E)$ образовано вкладами „верхних“ (лежащих на осях, ортогональных к оси приложения давления) долин, переходы из состояний $1 s(E)$ в условиях низких температур не наблюдаются. Положение наблюдаемой линии не изменилось и совпадает со значением $\sim 38$ мэВ, полученным из спектров образца без деформации [6] (рис. 1,b). Это означает, что данная линия связана с переходом из состояния $1 s\left(B_{2}\right)$ (нижняя компонента $1 s\left(T_{2}\right)$ при давлении в направлении [001]) в состояние $2 p_{0}$. Отметим, что наблюдается также линия вблизи 43.5 мэВ, соответствующая переходу $1 s\left(B_{2}\right) \rightarrow 2 p_{ \pm}$(на рисунке не указана).

Измерения в недеформированном образце кремния [6], в отличие от случая одноосно-сжатых образцов, показали наличие линий, которые связаны с оптическими переходами из обоих состояний $1 s(E)$ и $1 s\left(T_{2}\right)$ отщепленной группы в $2 p_{ \pm}$состояние. Полученные значения энергий для $1 s(E)$ и $1 s\left(T_{2}\right)$ состояний составили 47.5 и 49.9 мэВ соответственно. Стоило ожидать существования двух „лестниц“ переходов, связанных как с пара-, так и ортосостояниями гелиеподобного донора. Однако при конечном отношении

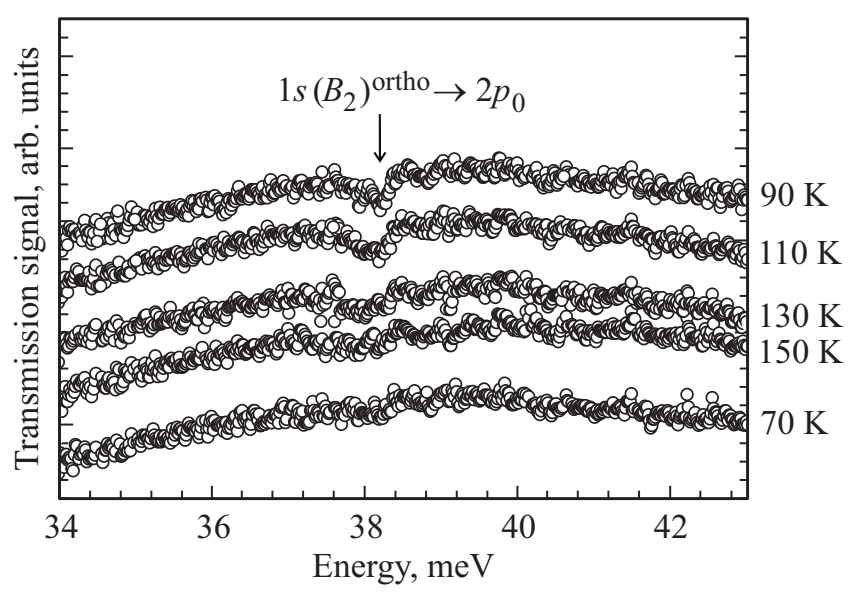

Pис. 2. Спектры пропускания нейтрального $\mathrm{Mg}$ в кремнии при различных температурах и одноосной деформации $S>2$ кбар вдоль направления [001]. 


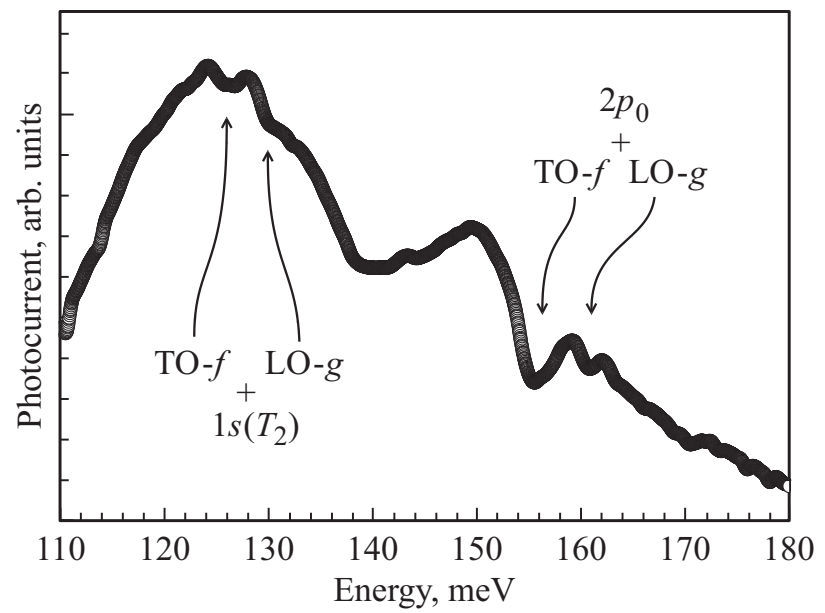

Рис. 3. Спектр фотопроводимости $\mathrm{Si}: \mathrm{Mg}$ при температуре жидкого гелия. Показаны резонансы Фано для состояний $2 p_{0}$ и $1 s\left(T_{2}\right)$. Энергия $1 s\left(T_{2}\right)$ составляет $41 \pm 1$ мэВ.

„сигнал/шум“ видны лишь наиболее сильные линии. Ввиду того что ортосостояния вырождены по спину трехкратно, следует отнести полученное положение состояний $1 s\left(B_{2}\right) / 1 s\left(T_{2}\right)$ и $1 s(E)$ к спиновому триплету, а сами состояния обозначить $1 s(E)^{\text {ortho }}$ и $1 s\left(T_{2}\right)^{\text {ortho }}$. Напротив, природа резонансов Фано с учетом малой величины спин-орбитального взаимодействия позволяет наблюдать именно парасостояния (рис. $1, c$ ). Наличие резонансов Фано показали измерения спектров фотопроводимости (рис. 3). Результаты эксперимента были проинтерпретированы по аналогии с подобными измерениями в донорах VI группы в кремнии [7], что позволило дополнить схему уровней магния данными об отщепленных парасостояниях $1 s$. Резонансы, показанные на рис. 3 в диапазоне энергий кванта излучения $125-135$ мэВ, согласно интерпретации, связаны с состоянием $1 s\left(T_{2}\right)^{\text {para }}$, энергия которого составляет $41 \mathrm{мэВ.} \mathrm{Учитывая} \mathrm{то,} \mathrm{что}$ разница энергий между $1 s(E)^{\text {ortho }}$ и $1 s\left(T_{2}\right)^{\text {ortho }}$, согласно спектроскопическим измерениям, составляет 2.4 мэВ и что ожидаемая разница энергий между $1 s(E)^{\text {para }}$ и $1 s\left(T_{2}\right)^{\text {para }}$ должна иметь приблизительно такое же значение, можно дать оценку энергии состояния $1 s(E)^{\text {para }}$ как $38.6 \pm 1$ мэВ. Подытоживая полученные экспериментальные результаты можно заключить, что энергии спинтриплетных состояний $1 s(E)^{\text {ortho }}$ и $1 s\left(T_{2}\right)^{\text {ortho }} \sim 47.5$ мэВ и $\sim 49.9$ мэВ, а энергии спин-синглетных состояний $1 s(E)^{\text {para }}$ и $1 s\left(T_{2}\right)^{\text {para }} \sim 38.6 \pm 1$ мэВ и $\sim 41$ мэВ соответственно (см. таблицу). При полученных положениях уровней энергии состояний $1 s(E)^{\text {para }}$ и $1 s\left(T_{2}\right)^{\text {para }}$ быстрая релаксация $2 p_{0}$ уже не может определяться переходами в группу состояний $1 s\left(E, T_{2}\right)$, как в донорах V группы, так как в этом случае получаемое теоретическое значение темпа релаксации состояния $2 p_{0}$ намного меньше $10^{10} \mathrm{c}^{-1}$. По-видимому, скорость его релаксации обусловлена переходами в состояние $2 s$, энергия которого в двухзарядных центрах должна быть несколько меньше, чем энергия $2 p_{0}$. Положения уров-
Положение уровней отщепленной группы состояний $1 s$

\begin{tabular}{l|c|c|c|c}
\hline Состояние & $1 s(E)^{\text {ortho }}$ & $1 s\left(T_{2}\right)^{\text {ortho }}$ & $1 s(E)^{\text {para }}$ & $1 s\left(T_{2}\right)^{\text {para }}$ \\
\hline Энергия, мэВ & 47.5 & 49.9 & $38.6( \pm 1)$ & 41
\end{tabular}

ней, отвечающих состояниям $2 s(E), 2 s\left(T_{2}\right)$ и $2 s\left(A_{1}\right)$ донора магния в кремнии, неизвестны, но, проводя аналогию с другими двухзарядными центрами в кремнии (доноры VI группы), можно предположить, что энергии состояний $2 s\left(E, T_{2}\right)$ близки к энергии состояния $2 p_{0}$, а энергия $2 s\left(A_{1}\right)$ из-за потенциала центральной ячейки сдвинута ниже. Так, в донорах VI группы $(\mathrm{S}, \mathrm{Se}, \mathrm{Te})$ энергии состояний $2 s\left(A_{1}\right)$ имеют значения в диапазоне $15-18$ мэВ.

Для анализа возможности реализации различных лазерных схем, основываясь на полученных в эксперименте значениях энергий $1 s(E)^{\text {para }}$ и $1 s\left(T_{2}\right)^{\text {para }}$ состояний, был произведен расчет темпов „распада“ состояний $2 p_{0}$ и $2 s\left(A_{1}\right)$ в зависимости от энергии последнего. Результат расчета представлен на рис. 4. Темп внутридолинного перехода $2 p_{0} \rightarrow 2 s$ рассчитан в однодолинном приближении, где отличие темпов переходов связано только с отличием в энергиях соответствующего $2 s$ состояния, поэтому кривая для скорости перехода $2 p_{0} \rightarrow 2 s$ применима для любого $2 s$ состояния: $2 s(E), 2 s\left(T_{2}\right), 2 s\left(A_{1}\right)$.

Результаты вычислений показывают, что скорости релаксации состояний достигают довольно существенных значений: темп релаксации состояния $2 p_{0}$ имеет значение $\sim 2 \cdot 10^{10} \mathrm{c}^{-1}$ при положении уровня $2 s$ в диапазоне энергий от 17 до $22 \mathrm{мэB}$, что соответствует ожидаемому диапазону значений энергии $2 s\left(A_{1}\right)$ состояния. Релаксация же состояния $2 s$ в этом же интервале его энергий $\sim(2-5) \cdot 10^{10} \mathrm{c}^{-1}$. Таким образом, скорость релаксации состояния $2 s\left(A_{1}\right)$ может быть достаточной, чтобы рассчитывать на возможность наблюдения эффек-

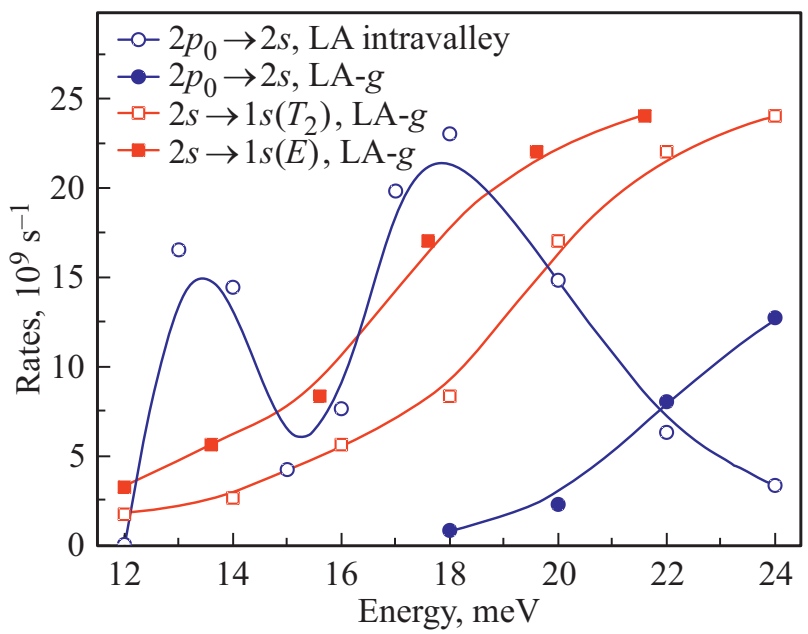

Рис. 4. Темпы релаксации уровней нейтрального донора $\mathrm{Mg}$ в кремнии в зависимости от положения уровня $2 s$. Для определенности энергия состояния $1 s(E)$ принималась равной 38.6 мэВ. 
та вынужденного комбинационного рассеяния света на переходе $1 s\left(A_{1}\right) \rightarrow 2 s\left(A_{1}\right)$ (рис. 1). Кроме того, такой оптический переход очень эффективен: по оценкам, его матричный элемент превосходит матричный элемент перехода $2 p_{0} \rightarrow 1 s$ (на котором развивается стимулированное излучение в донорах $\mathrm{V}$ группы) в $\sim 4$ раза, т.е. $\left\langle\Psi_{1 s}|x| \Psi_{2 p 0}\right\rangle /\left\langle\Psi_{2 s}|x| \Psi_{2 p 0}\right\rangle \approx 4$. Другая возможность реализация вынужденного комбинационного рассеяния света в магнии на переходе $1 s\left(A_{1}\right) \rightarrow 1 s(E)$, как это было продемонстрировано для доноров V группы в кремнии [1]. Напротив, реализация схемы с инверсией населенности на переходах $2 p \rightarrow 1 s$ видится трудно реализуемой ввиду достаточно быстрых времен релаксации состояний $2 p$ магния, по крайней мере, при малых деформациях и случая фотоионизации.

\section{4. Заключение}

С помощью спектроскопии пропускания и фотопроводимости обнаружены пара- и ортосостояния $1 s$ нейтрального донора магния. Измеренные значения отличаются от полученных ранее значений в работе [3], где предполагалось, что деформационная зависимость энергии основного состояния описывается формулой, полученной для водородоподобных доноров. В случае большого химического сдвига одноэлектронное приближение хорошо описывает возбужденные состояния гелиоподобных центров. Для доноров магния такой подход не точен.

Используя полученные значения энергии состояний $1 s\left(T_{2}, E\right)$ и варьируя положение уровней $2 s$, сделаны оценки возможных темпов релаксации как указанных состояний, так и уровня $2 p_{0}$. На основе полученных данных сделан вывод о возможности реализации механизма вынужденного рассеяния света на переходах $1 s\left(A_{1}\right) \rightarrow 2 s\left(A_{1}\right)$ и $1 s\left(A_{1}\right) \rightarrow 1 s(E)$.

\section{Финансирование работы}

Работа поддержана в рамках проекта Российского фонда фундаментальных исследований (19-02-00979) и совместного российско-германского проекта (№ 389056032 и 18-502-12077-ННИО). Использовано оборудование ЦКП ИФМ РАН. Работа выполнена в рамках государственного задания ИФМ РАН на 2019 г. 0035-2019-0020-C-01.

\section{Конфликт интересов}

Авторы заявляют, что у них нет конфликта интересов.

\section{Список литературы}

[1] S.G. Pavlov, R.Kh. Zhukavin, V.N. Shastin, H.-W. Hübers. Phys. Status Solidi B, 250, 9 (2013).

[2] H.G. Grimmeiss, E. Janzén, K. Larsson. Phys. Rev. B, 25, 2627 (1982).
[3] L.T. Ho, A.K. Ramdas. Phys. Rev. B, 5, 462 (1972).

[4] A.K. Ramdas, S. Rodriguez. Rep. Prog. Phys., 44, 1297 (1981).

[5] Yu.A. Astrov, V.B. Shuman, L.M. Portsel, N.A. Lodygin, S.G. Pavlov, N.V. Abrosimov, V.N. Shastin, H.-W. Hübers. Phys. Status Solidi A, 214, 1700192 (2017).

[6] S.G. Pavlov, N.V. Abrosimov, V.B. Shuman, H.L.M. Portsel, A.N. Lodygin, Yu.A. Astrov, R.Kh. Zhukavin, V.N. Stastin, K. Irmscher, A. Pohl, H.-W. Hübers. Phys. Status Solidi B, 255, 1800514 (2018).

[7] E. Janzén, G. Grossmann, R. Stedman, H.G. Grimmeiss. Phys. Rev. B, 31, 8000 (1985).

Редактор А.Н. Смирнов

\section{The chemical shift and the energy of the exchange interaction of the $1 s$ states of magnesium donors in silicon. Possibility of stimulated emission}

V.N. Shastin ${ }^{1}$, R.Kh. Zhukavin ${ }^{1}$, K.A. Kovalevsky' ${ }^{1}$, V.V. Tsyplenkov ${ }^{1}$, V.V. Rumyantsev ${ }^{1}$, D.V. Shengurov ${ }^{1}$, S.G. Pavlov' , V.B. Shuman ${ }^{3}$, L.M. Portsel ${ }^{3}$, A.N. Lodygin ${ }^{3}$, Yu.A. Astrov ${ }^{3}$, N.V. Abrosimov ${ }^{4}$, J.M. Klopf ${ }^{5}$, H.-W. Hübers ${ }^{2,6}$

${ }^{1}$ Institute for Physics of Microstructures, Russian Academy of Sciences, 607680 Nizhny Novgorod, Russia

${ }^{2}$ Institute of Optical Sensor Systems, German Aerospace Center (DLR), 12489 Berlin, Germany

${ }^{3}$ loffe Institute, 194021 St. Petersburg, Russia

${ }^{4}$ Leibniz-Institut für Kristallzächtung (IKZ), 12489 Berlin, Germany

${ }^{5}$ Helmholz-Zentrum Dresden-Rossendorf, 01328 Dresden, Germany

${ }^{6}$ Department of Physics, Humboldt-Universiät zu Berlin, 12489 Berlin, Germany

Abstract The results of experiments on observation of split off $1 s$ states that allow to determine the chemical shift and energy of exchange interaction of neutral magnesium donor in silicon are presented. The energies of $1 s(E), 1 s\left(T_{2}\right)$, as well as $2 s\left(A_{1}\right)$ para states, determine the possibility of population inversion and particular mechanism of stimulated Raman scattering. The energy of $1 s\left(T_{2}\right)$ para state was determined by Fano resonances in $\mathrm{Si}: \mathrm{Mg}$ photoconductivity spectra at $T=4 \mathrm{~K}$, while energies of $1 s\left(T_{2}\right)$, $1 s(E)$ ortho states were found based on transmission spectra at elevated temperatures. The relaxation rates have been estimated and possible stimulated emission mechanisms have been analyzed based on obtained experimental data. 